Journal of Experimental and Clinical Medicine https://dergipark.org.tr/omujecm

Case Report

J Exp Clin Med

2021; 38(3): 376-378

doi: 10.52142 omujecm.38.3.31

\title{
Gastrointestinal bleeding due to giant gastric bezoar
}

\section{Fatih ÇALIŞKAN ${ }^{1, *}$ (i), İsmail Alper TARIM ${ }^{2}$ (D), Hızır Ufuk AKDEMİR ${ }^{3}$ (i), Sultan ÇALIŞKAN ${ }^{4}$ (i) Bülent GÜNGÖRER ${ }^{1}$ (i), Hatice ÖLGER UZUNER ${ }^{5}$ (i), Kağan KARABULUT 3 (i)}

\author{
${ }^{1}$ Department of Emergency Medicine, Faculty of Medicine, Ondokuz Mayis University, Samsun, Turkey \\ ${ }^{2}$ Department of General Surgery, Faculty of Medicine, Ondokuz Mayıs University, Samsun, Turkey \\ ${ }^{3}$ Department of Surgical Pathology, Faculty of Medicine, Ondokuz Mayıs University, Samsun, Turkey \\ ${ }^{4}$ Clinic of Emergency Medicine, Ankara Bilkent City Hospital, Ankara, Turkey \\ ${ }^{5}$ Clinic of Surgical Pathology, Samsun Training and Research Hospital, Samsun, Turkey
}

\begin{tabular}{l}
\hline Received: 04.12.2019 • Accepted/Published Online: 14.02 .2021 \\
Abstract \\
Gastric bezoars occur in the stomach due to foreign body accumulation with an inability to pass through the pylorus. Major complications of \\
bezoars include intestinal obstruction, gastric ulcer, gastric perforation, and bleeding. Many gastric bezoars can often be treated conservatively. \\
Endoscopy has been commonly used in both the diagnosis and treatment of bezoars. We present a case that complained about abundant \\
gastrointestinal bleeding as well as abdominal distension and was successfully treated with emergency gastric surgery after the failure of bleed \\
control by endoscopic intervention due to giant gastric bezoar.
\end{tabular}

Keywords: bleeding, gastric bezoar, melena, phytobezoar

\section{Introduction}

Gastric bezoars occur in the stomach as a result of foreign body accumulation with inability to pass through the pylorus. Major complications of bezoars include intestinal obstruction, gastric ulcer, gastric perforation, and bleeding. The most frequently detected bezoar type is phytobezoars. Foods that lead to their development are Persimmon, orange and other citrus fruits, pulp-rich flesh, and vegetables. Persimmon contains shibutal. When Shibutal reacts with acid in the stomach, an insoluble viscous material is formed. Especially in a stomach with reduced acid secretion and motility due to previous surgery, this viscous material can form a nucleus for bezoar formation. Inadequate oral digestion and chewing due to toothless or damaged tooth structure, decreased gastric acidity, and slowing of gastric emptying as a result of previous gastric surgery. In various series, the stomach surgery rates as high as $55.6 \%$ and $70 \%$ were reported (Kadian et al., 1978; Ersan et al., 2005; Sumskiene et al., 2009; Khan et al., 2019).

"Gastroparesis diabeticorum" due to diabetic neuropathy has been shown as another factor that causes bezoars in the stomach. It has been suggested that diabetic neuropathy reduces gastric motility and delayed emptying of solid foods with vagotomy. Trichobezoar is seen in children with mental retardation and psychiatric patients. These patients may be paranoid, obsessive, or even with Down syndrome. A special type of trichobezoar is the Rapunzel syndrome. In these cases, apart from the hair mass in the stomach, there are hairballs starting from this gastric mass, passing the entire small intestine, and extending to the right colon. Petrified bodies of the stomach are hard bezoars that become hard and solid in the aqueous environment of the stomach. Most of them occur with the gammalax residue present in furniture polish. It was seen in six people in Nigeria who drink water mixed with industrial cement (Kadian et al., 1978; Ersan et al., 2005; Sumskiene et al., 2009; Khan et al., 2019).

Gastric phytobezoars can often be treated conservatively. Repeated doses of cellulase are the most effective proteolytic enzyme in dissolving stomach phytobezoars. Other proteolytic enzymes such as papain and acetylcysteine are not as effective as cellulase. Endoscopy has been used in both diagnosis and treatment of bezoars. Huge phytobezoars may need to be broken down and fragmented and the fragments removed individually. Sometimes, complete cleansing can be done in 34 sessions. There are studies stating that successful results are obtained from the use of "Electrohydraulic Lithotripsy" as an advanced form of endoscopic treatment and combined treatment with cellulase / cystein / metachopramide trio with endoscopic fragmentation (Kadian et al., 1978; Ersan et al., 2005; Sumskiene et al., 2009; Khan et al., 2019). In phytobezoars where conservative treatment is not sufficient, surgical treatment is required in all cement bezoars in most cases of trichobezoar. Surgical treatment can be done laparoscopically or open surgical methods (Kadian et al., 1978; 
Ersan et al., 2005; Sumskiene et al., 2009; Khan et al., 2019). We present a case who was admitted to the emergency department with abundant gastrointestinal bleeding and was successfully treated with emergency gastric surgery, because of gastrointestinal bleeding could not be controlled due to giant gastric bezoar after endoscopic intervention.

\section{Case report}

A 79-year-old man with complaints of abdominal pain, nausea, hematemesis and black stool was admitted to the emergency room of our university hospital. These symptoms appeared 15 hours before the admission. He had mentioned fullness in the stomach, a complaint lasting for about six months. Past medical history reported peptic ulcer disease with gastric ulcer perforation and surgical ulcer treatment with vagotomy about 20 years before the admission. Physical examination revealed gastrointestinal bleeding (GI) indicating melena. Abdomen was not tight, but painful in the epigastrium Blood analysis showed severe anema with a hemoglobin concentration of 5.4 $\mathrm{g} / \mathrm{L}$. Upper GI endoscopy showed a giant foreign body (dimensions; 12x6x4 cm) positioned longitudinally in the stomach and a huge ulcer coated exude formation. General surgery was recommended for the patient who could not be treated endoscopically by the gastroenterologist. Histological examination of biopsy specimens from ulcer and its adjacent area revealed a typical ulcer induced by pressure of bezoar (Fig. 1). There were defects in the mucosal integrity. There were typical changes of superficial ischemic damage, likely due to prolonged bezoar compression. Helicobacter pylori and intestinal metaplasia were not seen.

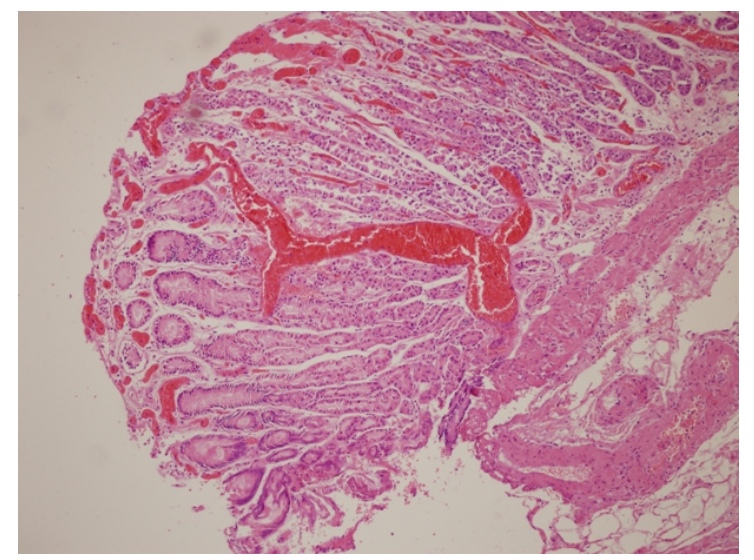

Fig. 1. Histological examination of biopsy specimens from ulcer and its adjacent area revealed a typical ulcer induced by pressure of bezoar

The patient was consulted to a general surgeon for surgical treatment. Abdominal computered tomography was administered. Abdominal CT showed a foreign body which had a contrast defect and a dimension of 12x6x4 cm (Fig. 2). There was an open anastomosis of gastrojejunostomy in the inferior of corpus. Although gastric bezoar was filled into the stomach, there was no obstruction of outlet to distal from the stomach according to CT. The giant bezoar was removed by surgical treatment (Figs. 3 and 4). After appropriate treatment, the patient who had a hemoglobin concentration of $11 \mathrm{~g} / \mathrm{L}$ was discharged healthily at the seventh day after surgery.

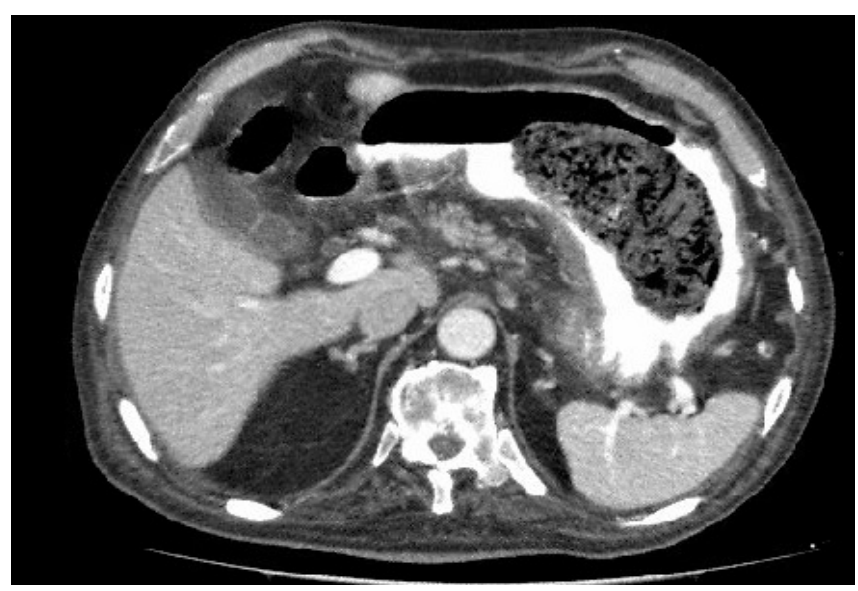

Fig. 2. Abdominal CT showed a foreign body which had a contrast defect and a dimension of $12 \times 6 \times 4 \mathrm{~cm}$

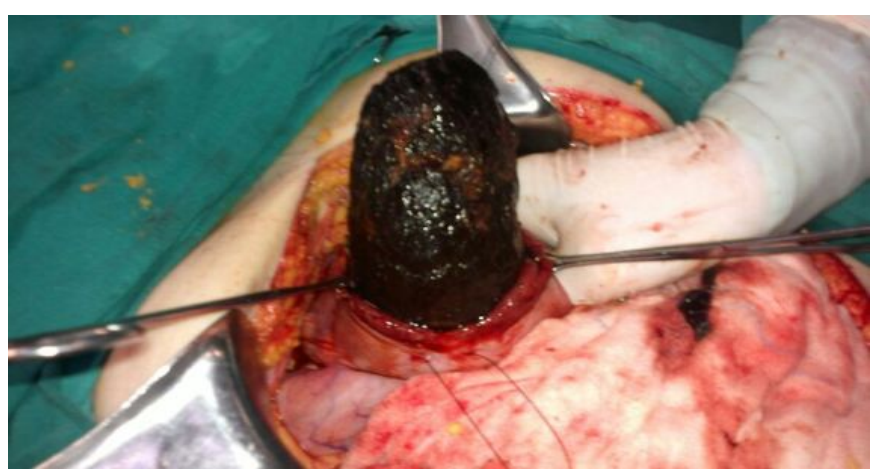

Fig. 3. Removal of bezoar by surgical treatment

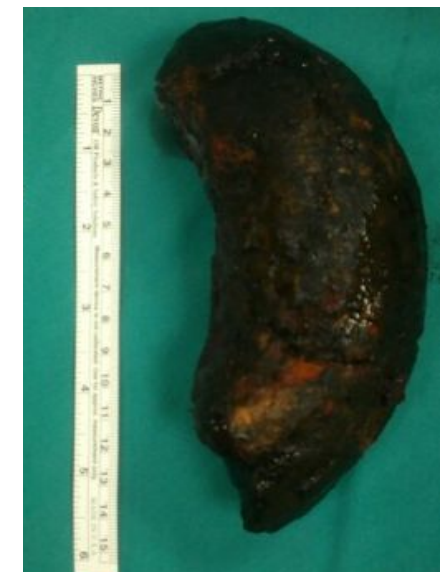

Fig. 4. Phytobezoar

\section{Discussion}

Gastric bezoars are rare (an estimated incidence of 0.3 percent on upper endoscopy), cause nonspecific symptoms, and are usually found incidentally in patients undergoing upper gastrointestinal endoscopy or imaging (Kadian et al., 1978). Our patient had complaints of abdominal pain, nausea and symptoms of gastrointestinal bleeding such as hematemesis and melena. Gastric bezoar was found firstly in GI endoscopy for GI bleeding and confirmed by abdominal CT.

Bezoars are classified according to their composition such as phytobezoars, trichobezoars, pharmacopoeias (e.g., extended release nifedipine, theophylline, enteric-coated aspirin, sodium alginate, and sucralfate) and other type (e.g., tissue paper, fungus, Styrofoam cups, cement, vinyl gloves, and, rarely, milk curd) (Sumskiene et al., 2009). The most 
common type of bezoars is phytobezoar and it was also seen in our patient. Several risk factors have been identified in patients with gastric bezoars. Risk factors include gastric dysmotility, gastric outlet obstruction, dehydration, use of anticholinergic agents and opiates and use of medications with an insoluble carrying vehicle (e.g., enteric-coated aspirin and nifedipine) (Krausz et al., 1986). Among patients with gastric bezoars, 70 to 94 percent have had gastric surgery and 54 to 80 percent have undergone vagotomy and pyloroplasty. Patients with gastroparesis appear to be at increased risk of formation of gastric bezoars due to impairment in the grinding mechanism of the stomach and the inter digestive migrating motor complex (Robles et al., 1994; White et al., 2003). Our patient had a history of previous gastric surgery and no medications.

The patients with bezoars are usually asymptomatic and symptom onset is insidious. The most common symptoms include abdominal pain, nausea, vomiting, early satiety, anorexia, and weight loss. Gastrointestinal bleeding due to concurrent gastric ulcers is a common presentation in patients who have previously undergone surgery. The ulcers may be due to peptic ulcer disease or pressure necrosis. Although many bezoars become quite large, gastric outlet obstruction is an uncommon presentation (DeBakey et al., 1938). He had abdominal pain, nausea, hematemesis and black stool. He had a history of peptic ulcer disease and previous gastric surgery. Upper gastrointestinal endoscopy is required to establish the diagnosis of a gastric bezoar and to obtain samples to determine its composition. Endoscopically, a gastric bezoar has the appearance of a dark brown, green, or black ball of amorphous material in the fundus or antrum of the stomach. In our patient, the gastric bezoar was identified by GI endoscopy. It was a dark brown (Figs. 3 and 4) (Gelrud and Gelrud, 2019).

The choice of therapy depends on the type of bezoar and the presence of underlying risk factors. The alternatives of treatment include chemical dissolution, endoscopic removal, adjuvant prokinetics (e.g., metoclopramide10 mg orally before meals and at bedtime) and surgery. Chemical dissolution involves administration of an agent to degrade the gastric bezoar. As compared with endoscopic therapy or surgery, chemical dissolution has the advantage of being noninvasive and inexpensive. However, a potential complication of chemical dissolution therapy is that partially dissolved bezoars may cause small bowel obstruction up to six weeks later. There are no randomized trials comparing these agents (e.g., CocaCola, Cellulase, Papain, Acetylcysteine) (Gelrud and Gelrud, 2019). In the literature, cases where chemical resolution was achieved by giving pineapple juice have been reported (Karabıçak et al., 2012). Large gastric bezoars, if pylorus is normal, usually are treated by endoscopy. The bezoar fragmentation can be performed with a large polypectomy snare, electrosurgical knife, lithotripter, drilling, endoscopic laser destruction, and Dormia basket or conventional surgery can be chosen (Sumskiene et al., 2009). Surgical removal should be reserved for patients who fail chemical dissolution and endoscopic therapy and for patients with complications including obstruction and significant bleeding. If a gastrotomy or enterotomy is performed to remove bezoars, the remainder of the small bowel and stomach should be examined to exclude other retained bezoars (Kadian et al., 1978; Sumskiene et al., 2009; Gelrud and Gelrud, 2019). Our patient had a severe anemia and symptoms of acute gastrointestinal bleeding. The GI endoscopy was failed to bleeding control and removal of bezoar. The most appropriate modality for our patient was surgical treatment. The bezoar was removed successfully and no complication was seen after surgery. He was discharged healthily at seventh day of surgery.

Large bezoars may be a cause of gastric ulcer and chronic gastrointestinal bleeding. Large gastric bezoars resulting from gastric dysmotility related to previous surgery have commonly been described, but complications such as ulcer caused by pressure of foreign body and gastrointestinal bleeding are especially rare. Patients with previous gastric surgery should be warned about this preventable complication and dietary advice should be given for these patients.

\section{Conflict of interest}

None to declare.

\section{Acknowledgments}

None to declare.

\section{References}

1. DeBakey, M., Oshner, A., 1938. Bezoars and concretions. Surgery. 4, 934.

2. Ersan, Y., Yavuz, N., Yüceyar, S., Çiçek, Y., Ergüney, S., Karataş, A., Belli, A., 2005. Gastric bezoars requiring surgical treatment. Cerrahpaşa J. Med. 36, 128-133.

3. Gelrud, D., Gelrud, M., 2019. Gastric Bezoars. In: Uptodate [online]. Accessed December 04, 2019.

4. Kadian, R.S., Rose, J.F., Mann, N.S., 1978. Gastric bezoars spontaneous resolution. Am. J. Gastroenterol. 70(1), 79.

5. Karabıçak, İ., Yürüker, S., Kesicioğlu, T., Çınar, H., Polat, C., Özen, N., 2012. Ananas juice for medical treatment of bezoars: Clinical experience with 4 patients. J. Exp. Clin. Med. 28, 55-58.

6. Khan, S., Jiang, K., Zhu, L.P., Khan, I.A., Ullah, K., Khan, S., Chen, X., Wang, B.M., 2019. Upper gastrointestinal manifestation of bezoars and the etiological factors: A literature review. Gastroenterol. Res. Pract. 2019, 1-13.

7. Krausz, M.M., Moriel, E.Z., Ayalon, A., Pode, D., Durst, A.L., 1986. Surgical aspects of gastrointestinal persimmon phytobezoar treatment. Am. J. Surg. 152, 526.

8. Robles, R., Parrilla, P., Escamilla, C., Lujan, J.A., Torralba, J.A., Liron, R., Moreno, A., 1994. Gastrointestinal bezoars. Br. J. Surg. 81, 1000 .

9. Sumskiene, J., Janciauskas, D., Pilkauskaite, G., Kristalnyj, V. Kupcinskas, L., 2009. An unusual case of bleeding from stomach due to a giant diospyrobezoar. Medicina (Kaunas). 45, 476-479.

10. White, N.B., Gibbs, K.E., Goodwin, A., Teixeira, J., 2003. Gastric bezoar complicating laparoscopic adjustable gastric banding, and review of literature. Obes. Surg. 13, 948. 